Reprod. Nutr. Dévelop., 1985, 25 (1 B), 197-210.

\title{
Cellularité des tissus adipeux chez le porcelet en fonction du niveau de l'apport énergétique du régime maternel et du nombre de jeunes par portée
}

\author{
Françoise DESNOYERS, M. ETIENNE (*), G. PASCAL (**), G. DURAND \\ Station de Recherches de Nutrition, \\ I.N.R.A., 78350 Jouy-en-Josas, France. \\ (*) Station de Recherches sur I'Elevage des Porcs, \\ I.N.R.A., Saint-Gilles 35590 L'Hermitage. \\ (**) Laboratoire des Sciences de la Consommation, \\ I.N.R.A., 78350 Jouy-en-Josas.
}

Summary. Adipose tissue cellularity in the piglet and its relationship with the level of energy supply of the maternal diet and the number of piglets per litter.

Adipose tissue cellularity has been evaluated in piglets at birth and at weaning at 35 days in relation with the energy supply of the maternal diet during gestation (group B : $3000 \mathrm{KcalED} /$ day : group N ; $6500 \mathrm{KcalED} /$ day ; group H : $10000 \mathrm{Kcal} /$ day) and with the number of piglets per litter during suckling.

This approximation was obtained by simultaneously measuring piglet carcass triglyceride content and the mean diameter of the lipid inclusions of the fat cell population.

At birth there were no significant differences between $\mathrm{N}$ and $\mathrm{H}$ piglets. However, $\mathrm{B}$ piglets weighed less and had lower lipid stores than the piglets of the other two groups. This lower adiposity was due to the smaller number of fat cells (about - $25 \%$ ).

A weaning piglet weight and adiposity depended on both the energy supply of the maternal diet during gestation and the number of piglets per litter. These differences were due to the number of fat cells ; this number was usually greater when the maternal diet during gestation had a higher energy level and the number of piglets per litter was smaller.

Between birth and weaning the number of fat cells increased by a factor related with the number of piglets per litter ; this factor was 1.9 or 2.5 , depending on whether the sow suckled 4 or 8 piglets.

\section{Introduction.}

Les effets du niveau énergétique de la ration pendant la gestation et la période d'allaitement sur la cellularité des tissus adipeux des individus jeunes ont fait l'objet d'études assez nombreuses. Ces travaux ont été réalisés chez plusieurs espèces animales, Rat et Souris (Knittle et Hirsch, 1968 ; Hirsch et Han, 1969 ; Greenwood et Hirsch, 1974 ; Rakow et al., 1970), Porc (Lister et MacCance, 1967 ; Anderson, 1971 ; Anderson et Kauffman, 1973 ; Lee et Kauffman, 1974 ; 
Mersmann et al., 1975 ; Hood et Allen, 1977 ; Gurr et al., 1977 ; Desnoyers et al., 1980) ainsi que chez l'Homme (Hirsch et Knittle, 1970 ; Björntorp et al., 1971 ; Brook, 1972 ; Ravelli et al., 1976). Les résultats obtenus par ces auteurs peuvent être ainsi résumés: une surnutrition précoce entraîne un accroissement du nombre des cellules adipeuses (hyperplasie) facilitant l'apparition d'une obésité hyperplasique, plus difficile à traiter qu'une obésité hypertrophique due à la seule augmentation de la taille des cellules adipeuses.

Cependant, ces résultats, fondés sur des méthodologies qui ne permettent pas de comptabiliser l'ensemble de la population adipeuse, ont été critiqués (Gurr et Kirtland, 1978). C'est pourquoi dans ce travail, où nous étudions les effets du niveau énergétique de l'alimentation maternelle pendant la gestation et de l'alimentation lactée sur la cellularité des tissus adipeux du porcelet, nous avons utilisé une méthode permettant une plus juste estimation de l'ensemble des cellules adipeuses, y compris des préadipocytes. Le Porc a été choisi cómme animal expérimental d'une part en raison de l'intérêt zootechnique de l'espèce, d'autre part parce que chez lui, comme chez l'Homme, les tissus adipeux apparaissent à mi-gestation et que leur mise en place très progressive permet une étude histologique satisfaisante.

\section{Matériel et méthodes.}

Animaux. - Des truies primipares gravides Large-White de 120 à $130 \mathrm{~kg}$ de poids corporel ont été réparties en trois lots recevant chaque jour la même quantité de protéines, minéraux et vitamines mais des quantités d'énergie digestible différentes, considérées comme faible (Lot $\mathrm{B}, 3000 \mathrm{Kcal} E D$ ), normale (Lot N, $6500 \mathrm{Kcal}$ ED) ou élevée (Lot H, $10000 \mathrm{Kcal} \mathrm{ED).}$

Après la mise-bas, seules les portées comportant 8 à 10 porcelets ont été étudiées de manière à éliminer l'effet " taille de la portée ".

A l'intérieur de chacun des lots, les portées ont été égalisées soit à 8 , soit à 4 petits pour juger de l'influence des niveaux d'alimentation lactée sur le développement ultérieur des tissus adipeux déjà marqués par l'apport énergétique distribué à leur mère au cours de la gestation. Les porcelets en surnombre ont été sacrifiés pour analyse ; soit 11 dans le lot $\mathrm{B}, 10$ dans le lot $\mathrm{N}$ et 10 dans le lot $\mathrm{H}$.

L'apport alimentaire fourni à toutes les truies pendant la lactation a été de $4 \mathrm{~kg} /$ jour d'un aliment équilibré.

Au moment du sevrage des porcelets, 35 jours après la naissance, des animaux ont été sacrifiés pour analyse soit 11 dans le lot $B, 10$ dans le lot $N$ et 6 dans le lot $\mathrm{H}$.

Prélèvements et préparation des tissus. - Chez les porcelets nouveau-nés, les tissus adipeux ont été disséqués, aussi rapidement que possible après le sacrifice, aux niveaux suivants : cou, dos, croupe, épaule, régions ventrale et inguinale pour les tissus adipeux externes et régions périrénale et cardiaque par les tissus adipeux internes. Au sevrage, les tissus adipeux des mêmes localisations ont été prélevés, ainsi que le tissu adipeux intramusculaire qui n'apparaît chez le porc qu'après la naissance. Les échantillons destinés aux coupes semi-fines pour la 
microscopie photonique et aux coupes ultra-fines pour la microscopie électronique ont été traités selon les méthodes classiques. Les lipides totaux des carcasses ont été extraits selon la méthode de Folch et al. (1957), puis les triglycérides ont été séparés des phospholipides (Börgstrom, 1952).

Estimation de la cellularité. - L'estimation du nombre des cellules adipeuses est fondée sur le rapport entre le volume total des triglycérides appartenant aux tissus adipeux et le volume moyen de lipides par cellule de nature adipocytaire. On a estimé que les triglycérides appartenant en propre aux tissus adipeux correspondent à la différence entre les triglycérides totaux de la carcasse et ceux qui appartiennent normalement à l'ensemble des cellules (ceux-ci étant considérés équivalents à la moitié de la valeur des phospholipides).

La détermination du volume moyen des inclusions lipidiques a été faite en combinant les résultats que l'on obtient par des mesures micrométriques en microscopie photonique à ceux que fournit l'application de la méthode morphométrique en microscopie électronique (Weibel et Gomez, 1962), adaptée aux tissus adipeux (Desnoyers et al., 1980).

\section{Résultats.}

\section{A la naissance.}

Les résultats concernant le poids et l'adiposité des porcelets à la naissance ont été regroupés dans le tableau 1.

Les porcelets issus du lot $B$ (apport énergétique faible) ont un poids moyen de carcasse de $806 \mathrm{~g}$ significativement inférieur à celui des animaux issus du lot $\mathrm{N}$ (apport énergétique normal) pour lesquels il est de $1058 \mathrm{~g}$. On remarque qu'à ce stade de développement les animaux du lot $\mathrm{H}$ (apport énergétique élevé) ont un poids moyen de carcasse de $993 \mathrm{~g}$, inférieur à celui des porcelets du lot $\mathrm{N}$ mais significativement supérieur à celui des animaux du lot $B$.

\section{TABLEAU 1}

Porcelets à la naissance. Poids vif et adiposité selon l'apport énergétique du régime maternel pendant la gestation.

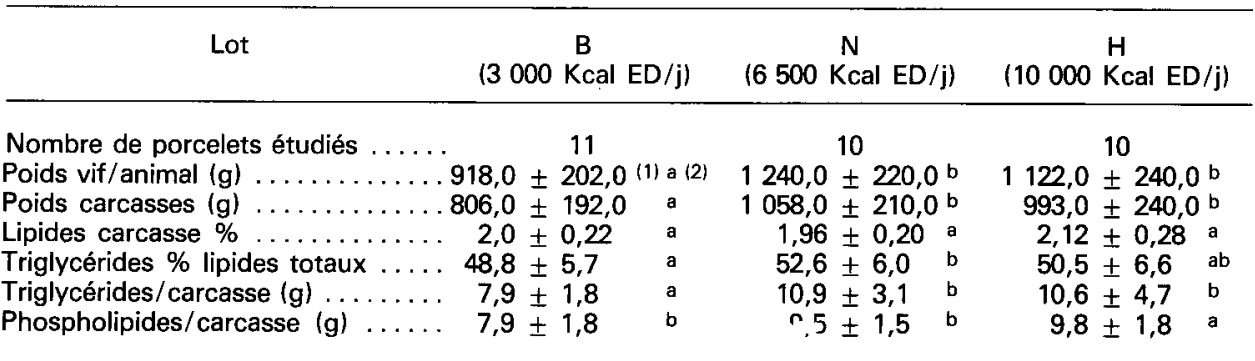

(1) Ecart-type; (2) Les valeurs affectées de lettres différentes sont significativement différentes au seuil de $5 \%$. 
Si la teneur en lipides des carcasses est comparable dans les trois lots, les lipides totaux des animaux du lot $\mathrm{N}$ sont cependant plus riches en triglycérides $(52,6 \%)$ que ceux des animaux du lot $\mathrm{B}(48,8 \%)$; les animaux du lot $\mathrm{H}$ renferment une quantité intermédiaire de triglycérides $(50,5 \%)$. Les carcasses des animaux des lots $\mathrm{N}$ et $\mathrm{H}$ contiennent plus de triglycérides et de phospholipides (respectivement $10,9 \mathrm{~g}, 10,6 \mathrm{~g}$ et $9,5 \mathrm{~g}, 9,8 \mathrm{~g}$ ) que celles des porcelets du lot B $(7,9 \mathrm{~g}$ et $7,9 \mathrm{~g})$.

Les résultats des mesures micrométriques en microscopie photonique et morphométriques en microscopie électronique ont été résumés dans le tableau 2.

On remarque que dans les trois lots, les cellules adipeuses des tissus adipeux externes ont des inclusions lipidiques dont le diamètre moyen mesuré est légèrement inférieur (environ $12,2 \mu \mathrm{m}$ ) à celui des cellules des tissus adipeux internes pour lesquels il varie de $12,9 \mu \mathrm{m}$ à $14,1 \mu \mathrm{m}$. Le diamètre moyen corrigé des inclusions lipidiques des adipocytes en microscopie photonique ne diffère pas sensiblement d'un lot à l'autre (aux environs de $16 \mu \mathrm{m}$ ). En microscopie électronique, les mesures morphométriques donnent également, pour les trois lots, des diamètres comparables compris entre $11,3 \mu \mathrm{m}$ et $12,5 \mu \mathrm{m}$.

Les différences observées entre les deux types de mesure tiennent au fait que les populations cellulaires étudiées ne sont pas absolument comparables dans les deux méthodes. En effet, à ce stade de développement, il existe un assez grand nombre de cellules adipeuses contenant des inclusions lipidiques de très faible diamètre qui ne peuvent être prises en compte dans les mesures en microscopie photonique alors que certaines cellules adipeuses de grande taille, mesurées en microscopie photonique, ne sont pas préservées lors de la préparation des coupes ultra-fines pour l'observation en microscopie électronique.

Le but de cette méthode étant de prendre en compte les préadipocytes (cellule de type adipeux sans inclusions lipidiques) dans le calcul du nombre des cellules adipeuses des porcelets, nous avons été amené à extrapoler le diamètre moyen corrigé des inclusions lipidiques à l'ensemble de la population adipeuse, c'est-à-dire les cellules adipeuses dont les inclusions lipidiques ont été mesurées et les préadipocytes.

Le pourcentage des cellules dont les inclusions lipidiques ne peuvent être mesurées et des préadipocytes varie de $34 \%$ à $40 \%$ dans le cas des observations en microscopie photonique, et de $25 \%$ a $30 \%$ dans le cas de cellules en microscopie électronique.

Les diamètres des inclusions lipidiques ainsi calculés sont, en microscopie photonique, semblables dans les lots $B$ et $N(9,7 \mu \mathrm{m})$ et légèrement supérieurs dans le lot $H(10,4 \mu \mathrm{m})$; en microscopie électronique, ils varient de $8,3 \mu \mathrm{m}$ pour le lot $\mathrm{H}$ à $8,5 \mu \mathrm{m}$ pour le lot $\mathrm{B}$ et à $9,3 \mu \mathrm{m}$ pour le lot $\mathrm{N}$. La moyenne arithmétique de ces diamètres calculés en microscopie photonique et en microscopie électronique donne les diamètres moyens définitifs des inclusions lipidiques de la population adipeuse sur lesquels seront basés les calculs nécessaires à l'évaluation du nombre des cellules de la population adipeuse des porcelets à la naissance (tabl. 3). 


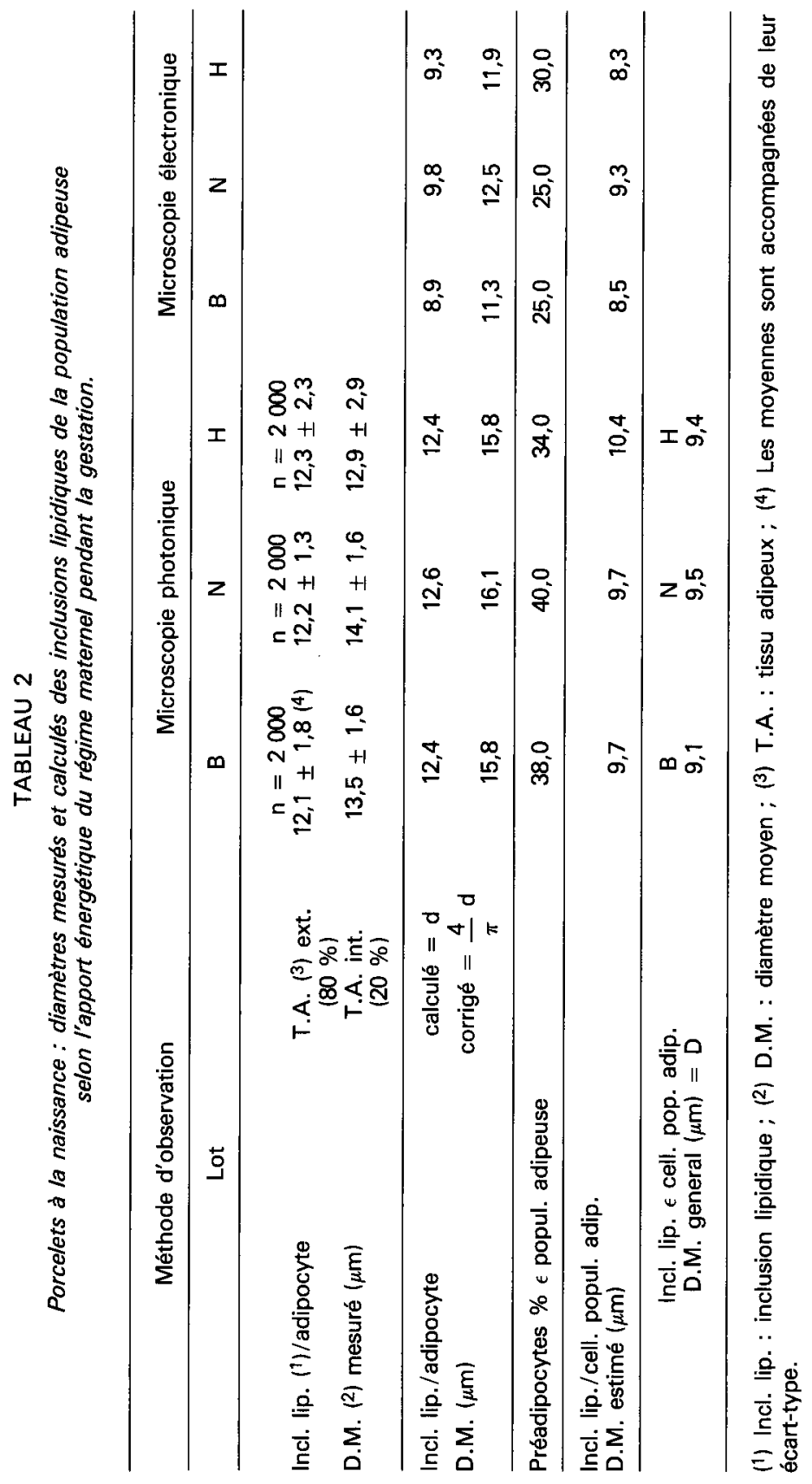


TABLEAU 3

Porcelets à la naissance : évaluation de la taille et du nombre des cellules de la population adipeuse par animal, selon le niveau de l'apport énergétique du régime maternel pendant la gestation.

\begin{tabular}{|c|c|c|c|c|}
\hline & Lot & B & $\mathrm{N}$ & $\mathrm{H}$ \\
\hline \multirow[t]{3}{*}{ Triglycérides T.A. ${ }^{(1)}(\mathrm{g})=\mathrm{P}$} & & 4,0 & 6,2 & 5,7 \\
\hline & $\left(10^{12} \mu \mathrm{m}^{3}\right)=V=\frac{P}{\text { densité }}$ & 4,4 & 6,8 & 6,2 \\
\hline & $\begin{array}{l}\text { Cell. pop. adip. } \\
\left(10^{2} \mu \mathrm{m}^{3}\right)=\mathrm{v}=\frac{\pi \mathrm{D}^{3}}{6}\end{array}$ & 394,0 & 449,0 & 435,0 \\
\hline \multicolumn{2}{|c|}{ Nb. cell. pop. adip. $(2) /$ porcelet $\left(\times 10^{9}\right)=\frac{V}{v}$} & 11,1 & 15,1 & 14,2 \\
\hline
\end{tabular}

(1) T.A. : tissu adipeux; (2) Nb. cell. pop. adip. : nombre de cellules de la population adipeuse.

L'estimation du nombre des cellules adipeuses par porcelet est effectuée en divisant le volume total des triglycérides propres aux tissus adipeux par le volume des triglycérides des cellules de la population adipeuse.

Le poids des triglycérides propres aux tissus adipeux a été calculé en soustrayant du poids des triglycérides extraits des carcasses la part de ceux appartenant à l'ensemble des cellules, que l'on estime correspondre à la moitié du poids des phospholipides. II résulte de cette estimation que les tissus adipeux des porcelets du lot $\mathrm{B}$ contiennent $4 \mathrm{~g}$ de triglycérides, ceux du lot $\mathrm{N} 6,2 \mathrm{~g}$ et ceux du lot $\mathrm{H}$ $5,7 \mathrm{~g}$.

Après caclul du volume des triglycérides des tissus adipeux en divisant leur poids par la densité de la trioléine $(0,915)$ et estimation du volume des triglycérides des inclusions lipidiques de la population adipeuse à partir du diamètre définitif des inclusions lipidiques, on peut évaluer le nombre des cellules de la population adipeuse pour chacun des lots.

Les porcelets des lots $\mathrm{N}$ et $\mathrm{H}$ ont un nombre de cellules assez comparable, respectivement $15,1 \times 10^{9}$ et $14,2 \times 19^{9}$, alors que l'on ne trouve que $11,1 \times 10^{9} \mathrm{cel}$ lules chez les porcelets du lot $B$.

\section{Au sevrage.}

Le tableau 4 regroupe les données concernant le poids et l'adiposité des porcelets au sevrage, 35 jours après la naissance, selon l'apport énergétique du régime maternel pendant la gestation et le nombre de jeunes par portée.

A ce stade de développement, on remarque qu'à l'intérieur de chacun des trois lots, les porcelets issus des portées faibles ont un poids de carcasse supérieur à celui des animaux issus des portées nombreuses. Comparé au poids moyen des carcasses des porcelets issus des portées nombreuses du lot $\mathrm{B}$, le poids moyen des carcasses des porcelets des deux autres lots $(\mathrm{N}$ et $\mathrm{H}$ ) élevés dans des portées de faible taille est significativement ou très significativement différent. La différence maximale est observée entre le poids moyen des carcasses des porcelets du lot $B$ (8) qui est de $5,4 \mathrm{~kg}$ et celui du lot $H$ (4) qui atteint $8,5 \mathrm{~kg}$. 


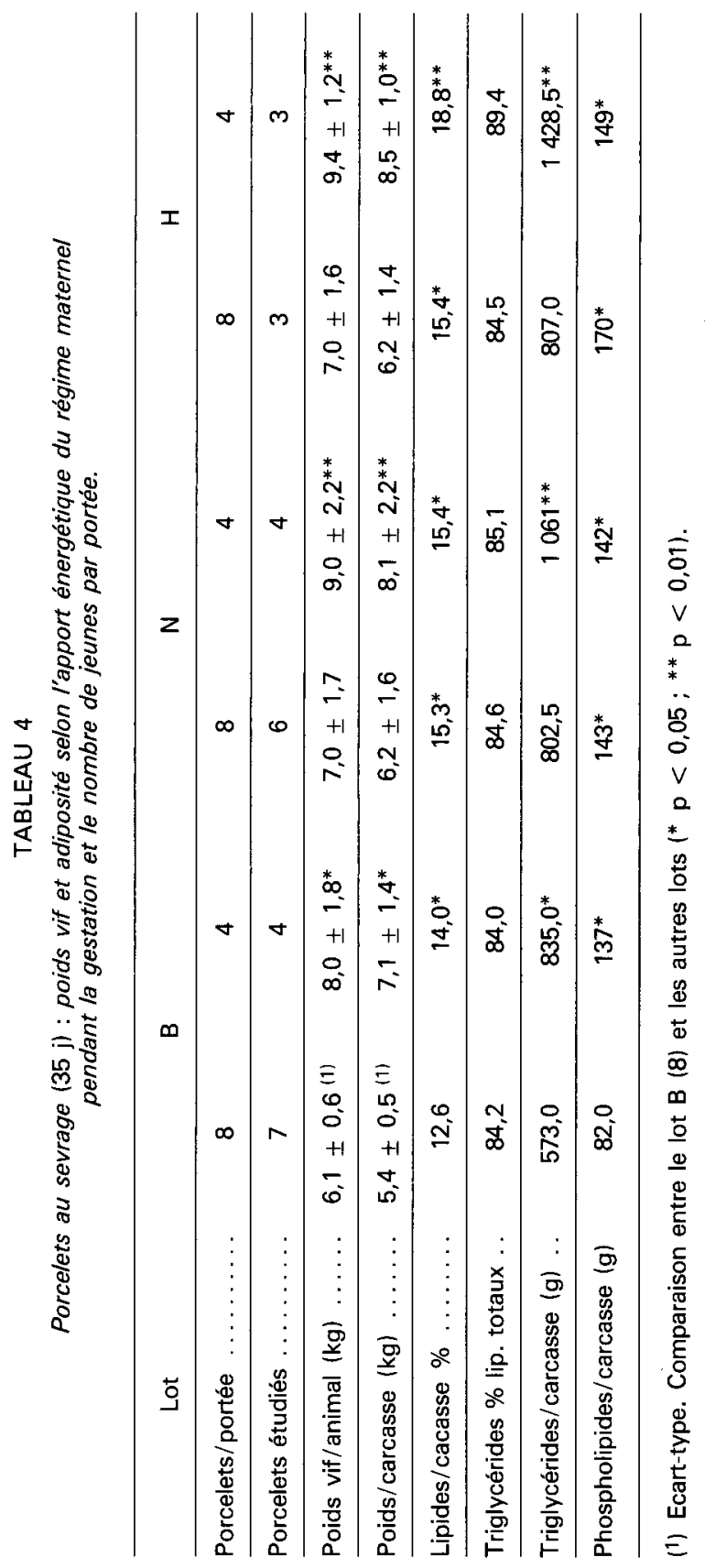


La teneur en lipides des carcasses des animaux des lots B (4), N (8) et (4) et H (8), qui varie de $14,0 \%$ à $15,4 \%$, est significativement différente de celle des porcelets du lot B (8) qui n'est que de 12,6\%. Dans le lot $H(4)$, cette teneur atteint $18,8 \%$, et diffère très significativement de celle des porcelets du lot $B(8)$.

La teneur en triglycérides des lipides totaux oscille entre $84,0 \%$ et $85,0 \%$ pour tous les lots quel que soit le nombre de jeunes par portée à l'exception du lot $H(4)$ pour lequel elle atteint $89,4 \%$. Le calcul du poids des triglycérides par carcasse permet d'observer que dans chacun des trois lots, les porcelets issus des portées de faible taille ont un poids de triglycérides plus élevé que ceux élevés dans des portées nombreuses et qu'en outre, il existe une différence significative entre le poids des triglycérides des porcelets du lot $B(4)$ avec celui des animaux du lot $B(8)$ et des différences hautement significatives avec ceux des lots $N(4)$ et $H$ (4). Le poids des phospholipides est sensiblement équivalent dans les trois lots pour les portées faibles et nombreuses et n'est que significativement inférieur pour les animaux du lot B (8).

Dans le tableau 5, nous avons rassemblé les données concernant les diamètres mesurés et calculés des inclusions lipidiques de la population adipeuse. Comme à la naissance, à l'intérieur de chaque lot, et quelle que soit l'importance de la portée, le diamètre des inclusions lipidiques est plus élevé dans les cellules des tissus adipeux externes que dans celles des tissus adipeux internes. Les inclusions lipidiques des tissus adipeux intramusculaires ont des diamètres encore plus faibles.

Lorsque l'on compare les lots entre eux, on s'aperçoit que les diamètres mesurés des inclusions des tissus adipeux externes et internes ont des valeurs approximativement comparables. En outre, les diamètres des inclusions lipidiques des tissus adipeux intramusculaires sont très constants quel que soit le lot et l'importance de la portée. Le diamètre moyen du contenu lipidique est ensuite calculé en tenant compte de la part relative de chaque catégorie de tissus adipeux dans l'organisme. Les résultats s'échelonnent de $42 \mu \mathrm{m}$ pour les animaux des lots $B(8)$ et $H(8)$ à $48 \mu \mathrm{m}$ pour ceux du lot $H(4)$. Les résultats ainsi obtenus correspondent au diamètre moyen des inclusions lipidiques sectionnées de façon aléatoire sur les coupes. L'application à ces données du coefficient de correction $4 / \pi$ permet d'estimer la valeur du diamètre moyen des inclusions lipidiques si les coupes avaient été effectuées dans le plan équatorial de ces inclusions. A ce stade du développement, les adipocytes entrent pour environ $70 \%$ dans le nombre des cellules de la population adipeuse. Il en résulte qu'en extrapolant à l'ensemble de la population adipeuse, le diamètre moyen estimé des inclusions lipidiques est ramené à des chiffres compris entre $32 \mu \mathrm{m}$ et $42 \mu \mathrm{m}$.

L'évaluation du nombre des cellules de la population adipeuse chez les porcelets au sevrage (tabl. 6) résulte, comme à la naissance, du rapport entre le volume des triglycérides propres aux tissus adipeux et celui des triglycérides des cellules de la population adipeuse. L'estimation du poids des triglycérides propres aux tissus adipeux s'effectue de la même façon qu'à la naissance : dans les 3 lots, les animaux issus de portées faibles ont un poids de triglycérides plus élevé que ceux appartenant à des portées nombreuses. 


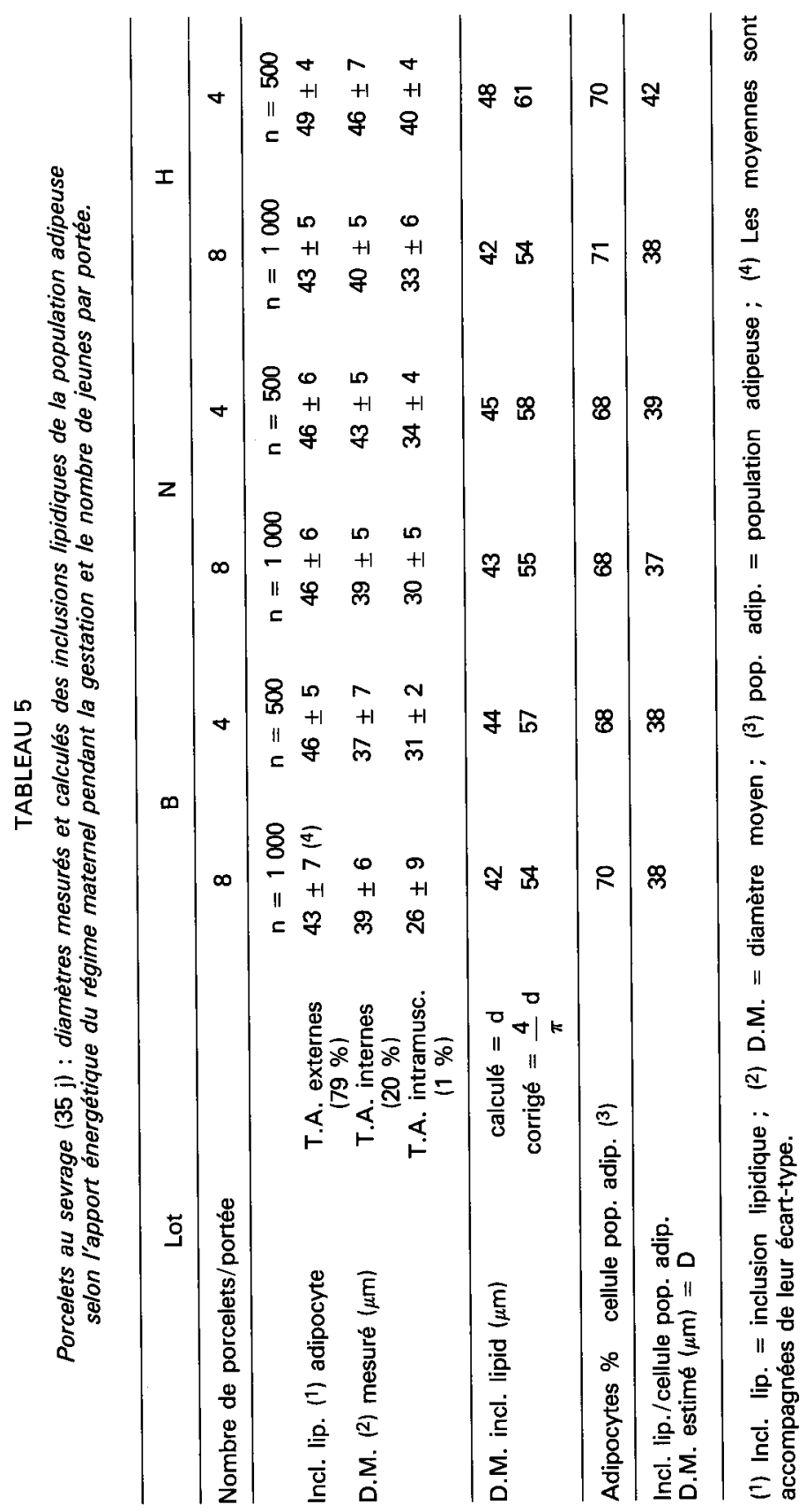




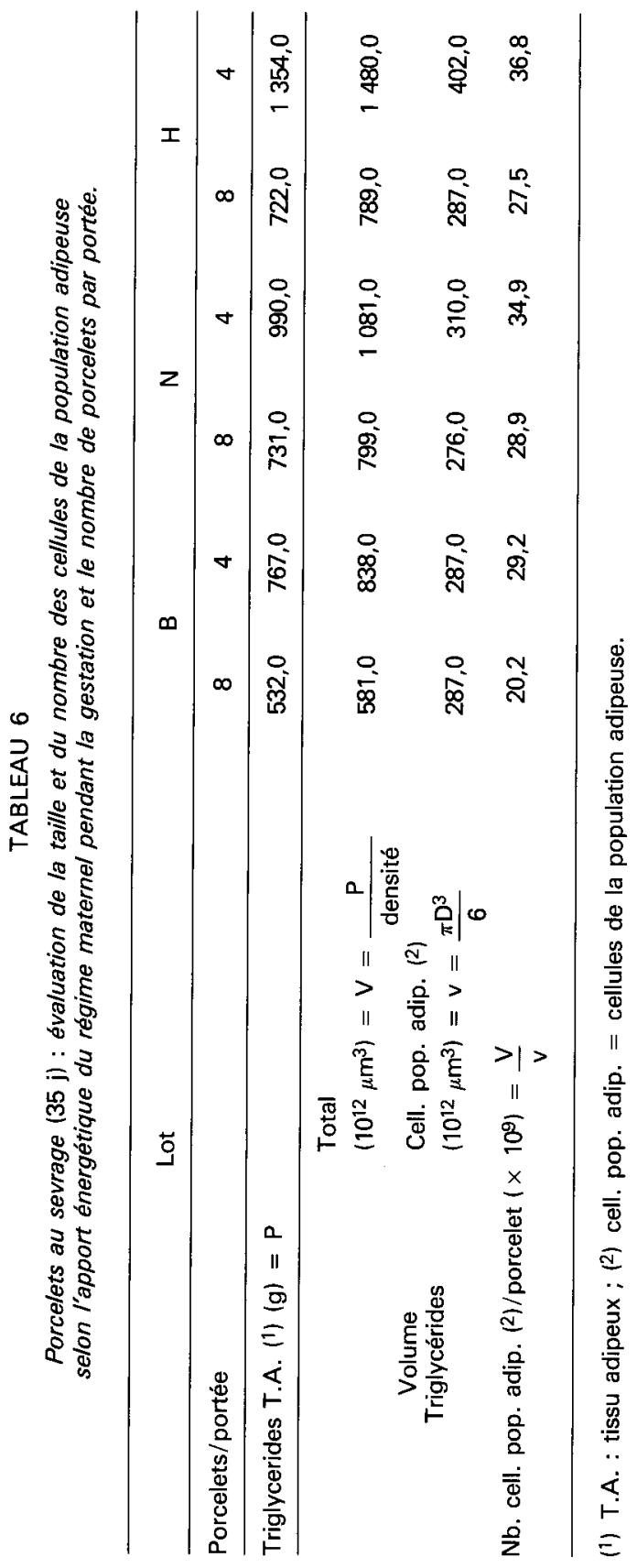


Après transformation du poids des triglycérides des tissus adipeux en volume et calcul du volume des inclusions lipidiques de la population adipeuse à partir du diamètre moyen estimé de ces inclusions, on évalue le nombre des cellules de la population adipeuse des porcelets en effectuant le rapport entre ces deux valeurs. Les résultats obtenus s'échelonnent entre $20,2 \times 10^{9}$ et $36,8 \times 10^{9}$ cellules. Les résultats obtenus pour les lots $B(4), N(8)$ sont comparables et situés aux environs de $29 \times 10^{9}$ cellules. Avec $34,9 \times 10^{9}$ cellules, le lot $\mathrm{N}(4)$ diffère très sensiblement, la différence maximale étant observée entre les lots $B(8)\left(20,2 \times 10^{9}\right.$ cellules) et $H(4)\left(36,8 \times 10^{9}\right.$ cellules $)$.

Lorsqu'on compare les résultats obtenus (tabl. 7) à la naissance et au sevrage, on constate que le diamètre des inclusions lipidiques par cellule de la population adipeuse est multiplié par un facteur d'environ 4,2, quel que soit le lot observé et indépendamment du nombre de jeunes allaités dans la portée. En ce qui concerne le nombre des cellules adipeuses, le facteur multiplicatif est lié au nombre de jeunes dans la portée et ceci quel que soit le lot. Ce facteur est d'environ 1,9 lorsque les mères élèvent 8 petits et passe à 2,5 lorsqu'elles n'en allaitent que 4.

\section{TABLEAU 7}

Evolution du diamètre et du nombre des cellules de la population adipeuse de la naissance au sevrage (35 j) chez le porcelet selon l'apport énergétique du régime maternel et le nombre de jeunes par portée.

\begin{tabular}{|c|c|c|c|c|c|c|c|}
\hline \multicolumn{2}{|c|}{ Lot } & \multicolumn{2}{|c|}{ B } & \multicolumn{2}{|c|}{$\mathbf{N}$} & \multicolumn{2}{|c|}{$\mathrm{H}$} \\
\hline & naissance & & & & & & \\
\hline \multirow[t]{2}{*}{ Diamètre $\left.{ }^{1}\right)(\mu \mathrm{m})$} & sevrage & $\begin{array}{l}\times 4,2 \\
38 \\
(8)(2)\end{array}$ & $\begin{array}{c}\times 4,2 \\
38 \\
(4)\end{array}$ & $\begin{array}{c}\times 4,0 \\
37 \\
(8)\end{array}$ & $\begin{array}{c}\times 4,1 \\
39 \\
(4)\end{array}$ & $\begin{array}{c}\times 4,0 \\
38 \\
(8)\end{array}$ & $\begin{array}{l}\times 4,5 \\
42 \\
\text { (4) }\end{array}$ \\
\hline & naissance & \multicolumn{2}{|c|}{11,1} & \multicolumn{2}{|c|}{15,1} & \multicolumn{2}{|c|}{ (8) $14,2^{\text {(4) }}$} \\
\hline Nombre $\left(\times 10^{9}\right)$ & sevrage & $\begin{array}{l}\times 1,8 \\
20,2 \\
(8)\end{array}$ & $\begin{array}{c}\times 2,6 \\
29,2 \\
(4)\end{array}$ & $\begin{array}{c}\times 1,9 \\
28,9 \\
(8)\end{array}$ & $\begin{array}{c}\times 2,3 \\
34,9 \\
(4)\end{array}$ & $\begin{array}{c}\times 1,9 \\
27,5 \\
(8)\end{array}$ & $\begin{array}{c}\times 2,6 \\
368 \\
(4)\end{array}$ \\
\hline
\end{tabular}

(1) Diamètre: diamètre moyen des inclusions lipidiques par cellule de la population adipeuse ;

(2) Nombre de porcelets par portée.

\section{Discussion et conclusions.}

L'étude de la cellularité des tissus adipeux chez le porcelet à la naissance et au sevrage ( 35 jours) a été effectuée en combinant les mesures de l'adiposité des porcelets à celles du contenu en triglycérides des cellules de l'ensemble de la population adipeuse, c'est-à-dire des adipocytes proprement dits et des préadipocytes. En effet, contrairement aux différentes méthodes utilisées précédemment par les auteurs (Rodbell, 1964 ; Goldrick, 1967 ; Hirsch et Gallian, 1968 ; Di Girolamo et al., 1971 ; Sjostrom et al., 1971) qui assimilent le contenu en triglycérides des cellules au volume cellulaire et ignorent la population préadipocytaire, nous avons utilisé une méthode de mesure en microscopie photonique associée à 
une méthode morphométrique en microscopie électronique permettant de prendre en compte uniquement le volume des triglycérides des cellules adipeuses et de dénombrer par comptage le pourcentage des préadipocytes par rapport à la population adipeuse globale. Cette technique semble mieux adaptée à l'étude de la cellularité des tissus adipeux chez les animaux jeunes.

L'ensemble des résultats concernant l'adiposité des porcelets montre qu'à la naissance l'accumulation des triglycérides dans la carcasse est faible, de l'ordre de $1 \%$ en poids et que la teneur en lipides des carcasses ne dépend pas du niveau de l'apport énergétique donné à la mère pendant la gestation. Les différences enregistrées sont liées aux différences de poids corporel, significativement plus faible dans le lot $B$.

Entre la naissance et le sevrage, on assiste à une accumulation importante de triglycérides puisque leur teneur passe de $1 \%$ à $9,4 \%$ pour le lot le plus maigre $(B, 8)$ et même $15,2 \%$ pour le lot le plus gras $(H, 4)$. Bien qu'il soit difficile d'exclure totalement l'effet dû au poids des animaux, celui des porcelets du lot B étant en moyenne plus faible que celui des porcelets des lots $N$ et $H$, quelle que soit la taille de la portée, c'est le niveau de l'apport énergétique de la mère pendant la gestation qui semble déterminer l'état d'engraissement des porcelets au sevrage.

En effet, et particulièrement lorsque les animaux sont placés à 4 sous la mère, ils sont d'autant plus riches en triglycérides de réserve à l'âge de 35 jours que les mères ont consommé plus d'énergie pendant la gestation. Toutes les truies ayant consommé la même quantité d'énergie pendant l'allaitement, on peut penser en accord avec Etienne (1979) que les différences observées s'expliquent par l'influence de l'alimentation durant la gestation sur la production laitière et en particulier sur la teneur du lait en lipides (Van Spaendonck, 1972) par le biais des réserves corporelles constituées par la truie avant la mise-bas.

A la naissance, si le contenu en triglycérides des cellules de la population adipeuse est comparable quel que soit le lot étudié, en revanche, le nombre des cellules adipeuses des porcelets du lot $\mathrm{B}$ est d'environ $25 \%$ inférieur à celui des animaux des lots $\mathrm{N}$ et $\mathrm{H}$. Ces résultats vont dans le sens des travaux de Ravelli et al. (1976), qui observent un moins grand nombre d'obèses chez les adolescents nés de mères ayant subi une restriction alimentaire pendant le dernier trimestre de leur grossesse. Chez ces deux espèces les tissus adipeux apparaissant de façon à peu près concomitante au cours de la gestation, on peut conclure qu'un apport très hypoénergétique à la mère pendant la période de mise en place des tissus adipeux a un effet réducteur sur le nombre des cellules de la population adipeuse chez le jeune.

Les nombreux travaux de restriction nutritionnelle effectués chez le Rat et la Souris (Knittle et Hirsch, 1968 ; Hirsch et Han, 1969 ; Rakow et al., 1970 ; Greenwood et Hirsch, 1974) tendent à montrer que chez ces deux espèces l'influence de la nutrition sur le nombre des cellules adipeuses est limitée aux trois premières semaines de la vie ; c'est-à-dire, là aussi, pendant la période d'installation des tissus adipeux qui, chez ces animaux, apparaissent après la naissance.

Au moment du sevrage, d'une part, seules les inclusions lipidiques des animaux du lot $H$ élevés à 4 sous la mère ont une taille supérieure à celle des inclu- 
sions des autres lots quelle que soit l'importance de la portée, d'autre part, à ce stade et quel que soit le lot observé, le nombre de cellules adipeuses s'accroît d'un facteur d'environ 2,5 lorsque les porcelets ont été élevés à 4 sous la mère. Enfin la comparaison inter-lots fait apparaître que les conditions nutritionnelles extrêmes [lot $B(8)$ et lot $H(4)$ ] entraînent des différences significatives dans le nombre des cellules de la population adipeuse des porcelets au sevrage. Toutes les truies allaitantes ayant reçu une même quantité d'un même aliment, on peut attribuer les différences observées, d'une part à la quantité d'énergie disponible pour chaque porcelet, variable selon que la portée est de 4 ou de 8 , d'autre part à la quantité différente d'énergie stockée par les mères au moment de la mise-bas.

Les résultats de cette expérience ne concordent pas avec les conclusions des travaux de Lee et al. (1973a et b) pour lesquels une restriction énergétique pendant les quatre semaines d'allaitement suivie d'un régime normal (ad libitum) pendant 120 jours n'entraîne aucune modification du nombre des adipocytes dans le tissu adipeux sous-cutané des porcs sacrifiés à l'âge de 148 jours. Au contraire, Martin et al. (1974) constatent que le nombre des cellules adipeuses du tissu adipeux périrénal est augmenté chez les porcelets élevés dans les portées de faible taille, ce qui rejoint nos observations.

10 Réunion du groupe Développement I.N.R.A., Rennes, 9-10 mai 1984.

\section{Références}

ANDERSON D. B., 1971. Cellular and enzymatic changes in porcine adipose tissue during growth. Thèse, Univ. Wisconsin, $147 \mathrm{p}$.

ANDERSON D. B., KAUFFMAN R. G., 1973. Cellular and enzymatic changes in porcine adipose tissue during growth. J. Lipid Res., 14, 160-168.

BJÖRNTORP P., BENGTSSON C., BLOHME G., JONSSON A., SJÖSTRÖM L., TIBBLIN E., TIBBLIN G., WIHELMSEN L., 1971. Adipose tissue fat cell size and number in relation to metabolism in randomly selected middle aged men and women. Metabolism, 20, 927-935.

BÖRGSTROM B., 1952. Investigation on lipid separation methods. Separation of phospholipids from neutral fat and fatty acids. Acta phys. scand., 25, 101-110.

BROOK C. G. D., 1972. Evidence for a sensitive period in adipose cell replication in man. Lancet, 2, 626-627.

DESNOYERS F., PASCAL G., ETIENNE M., VODOVAR N., 1980. Cellularity of adipose tissue in fetal pig. J. Lipid Res., 21, 301-308.

Di GIROLAMO M., MENDLINGER S., FERTIG J. W., 1971. A simple method to determine fat cell size and number in four mammalian species. Am. J. Physiol., 221, 850-858.

ETIENNE M., 1979. Influence de l'alimentation des truies gravides sur l'évolution des réserves corporelles maternelles et le développement de la portée. Ann. Biol. anim. Bioch. Biophys., 19, 289-302.

FOLCH J., LEES M., SLOANE-STANLEY G. H., 1957. A simple method for the isolation and purification of total lipids from animal tissues. J. biol. Chem., 226, 497-509.

GOLDRICK R. B., 1967. Morphological changes in adipocyte during fat deposition and mobilization. Amer. J. Physiol., 212, 777-782.

GREENWOOD M. R. C., HIRSCH J., 1974. Post-natal development of adipocyte cellularity in the normal rat. J. Lipid Res., 15, 474-483.

GURR M. I., KIRTLAND J., 1978. Adipose tissue cellularity : a review. 1. Techniques for studying cellularity. Int. J. Obesity, 2, 401-427. 
GURR M. I., KIRTLAND J., PHILLIP M., ROBINSON M. P., 1977. The consequence of early overnutrition for fat cell size and number : The pig as an experimental model for human obesity. Int. J. Obesity, 1, 151-170.

HIRSCH J., GALLIAN E., 1968. Methods for the determination of adipose cell size in man and animals. J. Lipid Res., 9, 110-119.

HIRSCH J., HAN P. W., 1969. Cellularity of rat adipose tissue : effect of growth, starvation and obesity. J. Lipid Res., 10, 77-82.

HIRSCH J., KNITTLE J. L., 1970. Cellularity of obese and non obese human adipose tissue. Fed. Proc. amer. Soc. exp. Biol., 29, 1516-1521.

HOOD R. L., ALLEN C. E., 1977. Cellularity of porcine adipose tissue effect of growth and adiposity. J. Lipid Res., 18, 275-284.

KNITTLE J. L., HIRSCH J., 1968. Effect of early nutrition on the development of rat epididymal fat pads : cellularity and metabolism. J. clin. Invest., 47, 2091-2098.

LEE Y. B., KAUFFMAN R. G., GRUMMER R. H., 1973a. Effect of early nutrition on the development of adipose tissue in the pig. I. Age constant basis. J. anim. Sci, 37, 1312-1318.

LEE Y. B., KAUFFMAN R. G., GRUMMER R. H., 1973b. Effect of early nutrition on the development of adipose tissue in the pig. II. Weight constant basis. J. anim. Sci., 37, 1319-1325.

LEE Y. B., KAUFFMAN R. G., 1974. Cellular and enzymatic changes with animal growth in porcine intramuscular adipose tissue. J. anim. Sci, 38, 532-538.

LISTER D., MCCANCE R. A., 1967. Severe undernutrition in growing and adult animals. The ultimate results of rehabilitation in pigs. Br. J. Nutr., 21, 787-799.

MARTIN R. J., EZEKWE M., HERBEIN J. H., SHERRITT G. W., GOBBLE J. L., ZIEGLER J. H., 1974. Effects of neonatal nutrition experiences on growth and development of the pig. $J$. anim. Sci., 39, 521-526.

MERSMANN H. J., GOODMAN J. R., BROWN L. J., 1975. Development of swine adipose tissue : morphology and chemical composition. J. Lipid Res., 16, 269-279.

RAKOW L., BENEKE G., MOHR W., BRAUCHLE I., 1970. Comparative morphological and chemical investigations on white and brown adipose tissue of the mouse after chronic starvation and refeeding. Beitr. Pathol., 141, 349-373.

RAVELLI G. P., STEIN Z. A., SUSSER M. V., 1976. Obesity in young man after famine exposure in utero and early infancy. New Engl. J. Med., 295, 349-353.

RODBELL M., 1964. Metabolism of isolated fat cells. I. Effects of hormones on glucose metabolism and lipolysis. J. biol. Chem., 239, 375-380.

SJÖSTROM L., BJÖRNTORP P., VRANA J., 1971. Microscopic fat cell size measurements on frozen cut adipose tissue in comparison with automatic determinations of osmium fixed fat cells. J. Lipid Res., 12, 521-530.

VAN SPAENDONCK R., 1972. Contribution to the study of the energy requirements of sows during gestation. Thèse, 252 p., Univ. Gand.

WEIBEL E. R., GOMEZ D. M., 1962. A principle for counting tissue structure on random sections. J. appl. Physiol., 17, 343-348. 